\title{
Les commerçants et les transporteurs dans l'approvisionnement vivrier et la distribution alimentaire à Douala (Cameroun)
}

\section{Hatcheu Emil Tchawe}

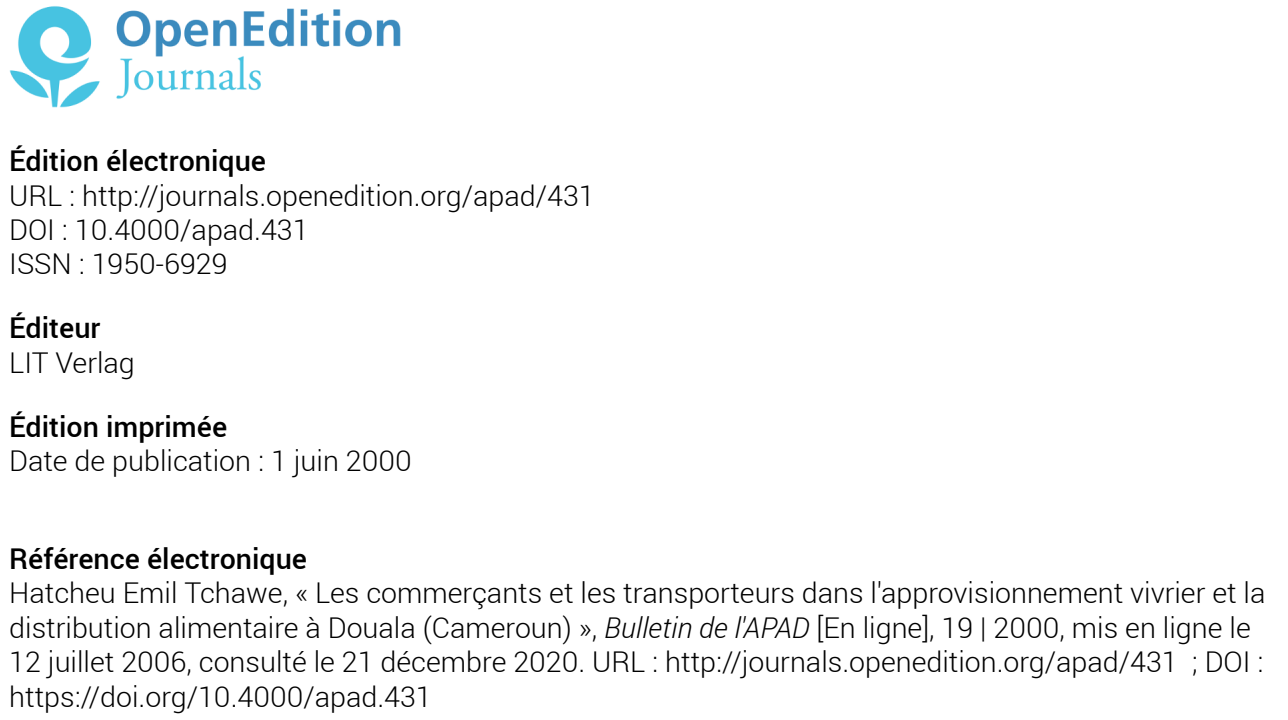

Ce document a été généré automatiquement le 21 décembre 2020.

Bulletin de l'APAD 


\title{
Les commerçants et les transporteurs dans l'approvisionnement vivrier et la distribution alimentaire à Douala (Cameroun)
}

\author{
Hatcheu Emil Tchawe
}

Selon les projections des Nations Unies, la population africaine devrait atteindre 1,6 milliard d'habitants en 2015. Cette forte croissance se traduira par l'augmentation du taux d'urbanisation dans la mesure où une bonne partie de cette population sera concentrée dans les grandes agglomérations urbaines. Ces prévisions relancent les inquiétudes sur la sécurité alimentaire durable des populations les plus démunies dans les pays en développement. Au Cameroun le schéma est identique. On note ici une augmentation rapide de la population citadine avec plusieurs pôles de croissance urbaine autour de certaines capitales provinciales (Garoua, Maroua, Bafoussam, Bamenda). Le taux d'urbanisation au Cameroun est passé de $28,5 \%$ en 1976 à $39 \%$ en 1987,45\% en 1995 et se situe aujourd'hui aux alentours de 50\%. Essentiellement rural et paysan au moment des indépendances il y a de cela moins d'un demi- siècle le Cameroun compte en ce début du troisième millénaire, 17 villes de plus de 50000 habitants, 9 villes de 100000 habitants et 5 villes de 200000 habitants. Par ailleurs, alors que le taux de croissance démographique annuel se maintient à $2,9 \%$, celui de la population urbaine est passé de 5\% entre 1964 et 1976, à 5,6\% entre 1976 et 1987, pour se stabiliser autour de 5,4\%. A côté de cette évolution globale, il importe d'accorder une attention particulière à la situation des deux grandes métropoles du pays que sont Douala, la capitale économique, et Yaoundé, la capitale politique avec un 1,5 million et 1,2 million d'habitants respectivement. Ces deux agglomérations concentrent $38,8 \%$ de la population urbaine nationale. 
2 Les conséquences de cette explosion urbaine sont entre autres, l'étalement spatial du périmètre urbain qui s'opère au détriment des espaces périphériques jusque là destinés à l'agriculture, la saturation de la plupart des infrastructures, l'intensification de la circulation, l'aggravation des difficultés de transport d'une part, l'exacerbation des conflits fonciers et le renchérissement des coûts d'approvisionnement vivrier d'autre part. Si la croissance urbaine suscite tant d'inquiétude, ce n'est pas seulement parce qu'il faut trouver des emplois pour les jeunes qui arrivent sur le marché du travail et construire de nouveaux logements pour des familles de plus en plus pauvres, mais, c'est surtout parce qu'il faut désormais nourrir convenablement cette population dont l'effectif augmente d'année en année.

"L'augmentation de la population urbaine donne une impulsion nouvelle à la commercialisation de la production vivrière dans la mesure il s'en suit une augmentation de la demande alimentaire. Cette demande peut être satisfaite par les importations, l'aide alimentaire ou par la production agricole intra et périurbaine ; mais elle l'est davantage par l'offre agricole rurale. En fonction des origines et des habitudes alimentaires des populations urbaines, elle portera sur une gamme plus ou moins variée de produits agricoles. Pour que l'approvisionnement des grandes métropoles en produits vivriers et la distribution alimentaire remplissent toutes les fonctions économiques et sociales souhaitées, il faut de véritables commerçants. un cadre juridique et des moyens de transport appropriés, enfin, un mode de fonctionnement et de gestion adéquats des marchés".

3 Pour assurer la paix sociale dans les villes, et dans une logique de parti pris pro-urbain, la stratégie des acteurs dominants (décideurs politiques et bailleurs de fonds internationaux) a consisté à maintenir les prix vivriers au niveau le plus bas possible. La sécurité alimentaire étant d'abord une question de demande, la mondialisation et les politiques d'ajustement structurel visent à pousser les pays en développement à une intégration rapide dans les grands courants d'échanges commerciaux. Pourtant l'ouverture des marchés nationaux, la suppression des barrières douanières et le triomphe de la vérité des prix auxquels les théoriciens de l'orthodoxie libérale poussent les pays en développement exposent les commerçants et les transports locaux à la concurrence des multinationales agro-alimentaires des pays industrialisés.

4 A plusieurs titres, les mesures prises dans le cadre des programmes d'ajustement structurel, qui fixent et orientent la politique macro-économique du Cameroun depuis 1988, ont eu des conséquences néfastes sur toutes les composantes de la sécurité alimentaire (disponibilité, stabilité et accessibilité). L'arrêt des subventions et la libéralisation totale du commerce des intrants affectent négativement la production agricole et l'offre de produits vivriers: le ravitaillement des centres urbains par les zones rurales est devenu plus compliqué et plus aléatoire du fait de l'augmentation des coûts de transport et des charges fiscales, obligeant les acteurs à modifier leurs comportements et leurs stratégies en matière d'approvisionnement et de distribution alimentaire dans les villes. Enfin, les compressions et les licenciements massifs du personnel dans les secteurs public et privé, la diminution des salaires et des revenus a eu des conséquences douloureuses sur le pouvoir d'achat et partant, sur la demande des ménages en denrées alimentaires.

5 Dans le cadre de cet exposé, nous essayons de montrer l'importance de l'approvisionnement vivrier dans les échanges villes/campagnes au Cameroun. A partir des flux alimentaires sur les différents marchés de la ville de Douala, nous nous efforcerons de montrer la dépendance de la capitale économique du Cameroun à l'égard de son hinterland. Nous esquisserons alors une typologie des ses acteurs. Grâce 
à la technique des récits de vie ou d'itinéraires nous avons amené quelques commerçants et transporteurs à nous dévoiler leurs stratégies de financement, leurs rapports avec les institutions financières, la nature de leurs relations avec les agents publics dans un environnement caractérisé par l'absence de la notion de service public et largement dominé par la parafiscalité pour ne pas dire la corruption. Auprès des détaillantes, nous avons essayé de comprendre la fonction et les logiques du "cadeau". Nous avons tenté de comprendre pourquoi sur nos marchés les revendeuses vendent plus en tas, en boîte, en panier ou en corbeille. Dans le contexte actuel de la mondialisation où les filières marchandes locales sont directement en concurrence avec les multinationales agro-alimentaires fortement soutenues par le capitalisme occidental, nous nous interrogerons enfin sur l'avenir des marchands africains dans l'approvisionnent et la distribution alimentaire de nos villes et des capitales occidentales où la diaspora devrait constituer des maillons essentiels des réseaux internationaux.

La dépendance de la capitale économique du Cameroun a l'égard de son hinterland

Pendant une vingtaine d'années (1970-1990), la problématique dominante de l'alimentation des villes africaines est surtout abordée en terme de relation ville/ campagne. On parle plus de "nourrir ou de ravitailler la ville" pour marquer à la fois l'importance accordée à la production agricole des zones rurales dans les politiques alimentaires, et "la bienveillance" de l'État providence dont la politique consiste à subventionner les importations alimentaires ou les intrants agricoles et à maintenir les prix des produits locaux à leurs niveaux les plus bas possible pour contenir les velléités revendicatrices des populations citadines maintenues en situation de dépendance soit des importations ou alors de l'arrière pays.

7 Cette vision, conforme à la politique d'autosuffisance alimentaire des États africains est fortement soutenue par les institutions internationales de développement comme la FAO ou la Banque mondiale. C'est que l'analyse des rapports villes/campagnes est largement influencée par les théories élaborées dans un contexte capitaliste où l'on assiste à une urbanisation des campagnes et à la mise en place d'un nouveau système de relations entre ces deux entités géographiques. En milieu urbain, l'agriculture est le plus souvent l'œuvre des femmes, qui considèrent ce travail comme relevant des tâches que la coutume leur réserve. Pour certaines, la culture est un palliatif contre une vie trop monotone en ville ou alors comme un complément à la ration versée par le chef de ménage. Pour d'autre enfin, l'alimentation de rue (beignet, pâte, bouillie, épis de mais grillés, igname, etc.) constitue une véritable activité génératrice de revenus. L'agriculture urbaine rapproche ainsi la ville du village. En même temps qu'elle constitue au cœur des grandes villes ou à leurs périphéries des poches de ruralité et de pauvreté. Comme le montre Schilter pour Lomé, l'agriculture urbaine et périurbaine est une des caractéristiques des villes africaines et la présence des cultures vivrières est un aspect très courant du paysage urbain. A l'intérieur même du périmètre urbain, de nombreuses concessions sont transformées en verger. L'association des plantes est générale ainsi que l'utilisation habile des pentes et des bas-fonds.

Loin d'être une sorte de survivance chez les néo-citadins, l'agriculture urbaine a retrouvé un regain de vitalité avec la crise économique, la baisse générale du pouvoir d'achat et les difficultés d'approvisionnement à partir des campagnes. Vennetier (1991) présente ainsi la ville comme le lieu de triomphe de l'économie monétaire. Pour y subsister le citadin est dans l'obligation de trouver du travail. Il conserve pendant un 
temps une situation de producteur agricole qui lui permet soit de couvrir ses propres besoins soit de pallier l'insuffisance de ses revenus. La ville exerce sur son hinterland, où elle puise hommes et biens dont elle a besoin, une puissante attraction.

9 En inscrivant l'analyse du phénomène urbain dans la hiérarchie et la dynamique spatiale des relations villes/campagnes, Courade (1985) estime que "le rôle que la ville joue dans le développement des campagnes est fonction des caractéristiques des villes dirigeantes et encadrantes, mais également des modes d'organisation des paysanneries et de la structuration des espaces ruraux". Il propose une typologie entre les sous-espaces ruraux, les périphéries rurales actives, les périphéries rurales passives et les sous-espaces marginaux rejoignant la différentiation faite entre l'agriculture intraou périurbaine, l'agriculture traditionnelle des petits producteurs, l'agriculture mixte vivrière et l'agriculture de rente. A la dichotomie ville/campagne se substitue une analyse des espaces hiérarchisés et des modes d'articulation entre les espaces urbains et ruraux. Les travaux de Goossens et al. (1994) et de Chaléard (1996) montrent le rôle déterminant de la campagne dans l'approvisionnement des villes africaines en proie à une forte croissance urbaine, malgré une la volonté pernicieuse de la mondialisation de rompre le cordon ombilical qui relie la ville à la campagne pour livrer la masse de consommateurs urbains au plus offrant: les multinationales occidentales. Si on peut percevoir la ville comme porteuse de déracinement et d'uniformisation face à l'espace rural, les rapports villes/campagnes s'inscrivent dans une histoire, une série de rapports sociaux, qui évoluent avec les techniques de contrôle de l'espace (moyens de transport, de communication, de coercition) (Courade, 1985). Et c'est à juste titre que Franqueville (1997) estime que de nombreuses villes dans les pays en développement vivent dans la hantise d'une rupture avec les zones d'approvisionnement de l'arrière-pays. Le cas de Douala au Cameroun en est une parfaite illustration.

10 Douala n'est pas la capitale du Cameroun, mais la ville la plus importante par sa population et son dynamisme économique. Avec un taux de croissance annuel de 5\%, elle devrait compter 3000000 d'habitants en 2020. Porte d'entrée maritime et aérienne du Cameroun et de certains pays enclavés de l'Afrique centrale (Tchad et République Centrafricaine notamment), Douala est le lieu de convergence de l'essentiel du réseau routier et ferroviaire camerounais. La ville accueille à elle seule trois quart de l'activité industrielle et commerciale du Cameroun. Malgré la crise et les politiques d'ajustement structurel qui ont suivi, le taux de chômage y est relativement bas (10\%), grâce au dynamisme du secteur informel où travaille $70 \%$ de la population active. Pendant longtemps, les populations autochtones ont été majoritaires à Douala, mais, depuis la fin de la seconde guerre mondiale, les allogènes venus de toutes les régions du Cameroun et de certains pays voisins (Tchad, RCA, Nigeria, Congo, Rwanda et Burundi) ont investi la ville à la recherche d'un emploi, pour des raisons scolaires ou pour fuir la guerre. Les courants de déversement les plus forts proviennent des régions les mieux desservies par la route ou par les rails (pays bamiléké et bassa). La zone d'influence de la métropole économique camerounaise comprend les régions suivantes: le Fako, le Nkam, la Sanaga Maritime, le Mungo et surtout la région des hautes terres de l'ouest (provinces de l'ouest et du nord ouest).

11 La vocation industrielle et commerciale de la ville de Douala liée à la présence du port se conjugue aux contraintes foncières, à la nature sablonneuse des sols et aux fortes précipitations pour réduire l'agriculture urbaine à un rôle marginal dans sa contribution à la sécurité alimentaire à Douala. Compte tenu des énormes possibilités 
qui existent dans le petit commerce d'une part, et de la forte demande en logement d'autre part, les terres sont en priorité affectées à la construction des maisons d'habitation. De plus, la fréquence des visites au village et l'existence de bonnes routes favorisent l'acheminement sur les marchés de Douala et dans les familles d'une gamme extrêmement variée de denrées (tubercules, céréales, feuille de banane, condiments etc.) qui entrent dans la préparation des plats de chaque groupe ethnique.

12 De plus, à l'inverse de la priorité accordée à l'augmentation de la production, la politique alimentaire camerounaise comporte des faiblesses notoires au triple niveau du stockage, de la transformation et de la distribution.. Il n' y a pas à proprement parler de stocks de sécurité alimentaire au Cameroun. L'Office Céréalier (structure dépendante du ministère en charge du développement industriel et commercial ) qui aurait dû jouer ce rôle, ne dispose que de structures de stockage de capacités réduite. Partout ailleurs, le système de stockage au Cameroun est réduit à des petits greniers que l'on peut trouver au niveau des ménages et qui sont malheureusement eux aussi en voie de disparition du fait de l'urbanisation des campagnes. De même, il n'existe aucun système formel de distribution des denrées alimentaires au Cameroun après la dissolution - au nom du désengagement de l'État et de la libéralisation - de la Mission de Développement des Cultures Vivrières (MIDEVIV).

On comprend alors que la capitale camerounaise dépende largement de l'arrière-pays pour son approvisionnement alimentaire. Et si toutes les régions du Cameroun y ont envoyé des ressortissants, l'importance des circuits d'approvisionnement et des réseaux marchands qui interviennent dans les différentes filières est fonction de l'effectif des différents groupes ethniques dans la population de la ville, de la survivance des habitudes traditionnelles de consommation au sein du groupe, de l'importance de la production agricole dans les différentes zones, du dynamisme économique du groupe, et enfin de la qualité des voies de communication entre ces régions et la métropole économique camerounaise. C'est pour toutes ces raisons que pour son approvisionnement vivrier la ville de Douala est largement tributaire de la zone du Mungo et, malgré son relatif éloignement, de la région des hautes terres de l'ouest du Cameroun.

14 La densité et la praticabilité du réseau routier et des pistes rurales sont des conditions essentielles pour la commercialisation des produits agricoles. Le désenclavement de certaines régions représente des atouts certains pour l'acheminement des produits sur les marchés vivriers, contribuant ainsi à la consolidation de la sécurité alimentaire des populations urbaines et des zones à risque ou à déficit vivrier.

15 A $200 \mathrm{Km}$ par route goudronnée, la région des hautes terre de l'ouest se situe en plein dans l'aire d'approvisionnement de Douala. Des grands travaux routiers (Douala-Limbé, Douala-Kekem) et ferroviaire (Douala-Edéa) ont renforcé les échanges entre Douala et son bassin d'approvisionnement vivrier. La construction et le bitumage des tronçons Dschang-Bamougoun et Bafoussam-Foumbot a parachevé la liaison avec les principaux marchés vivriers de l'ouest. Chez les Bamiléké qui animent l'essentiel de l'approvisionnement de Douala à partir de leur région d'origine et du Mungo qu'ils colonisèrent jadis, nous étudierons dans la suite de cette étude les logiques sociales qui se développent dans les pratiques commerciales.

Les logiques sociales dans les pratiques commerciales

16 La campagne bamiléké produit pour se nourrir et de plus en plus pour vendre; aucune zone de cette région ne vit en marge des échanges monétaires. Le marché traditionnel 
est l'élément essentiel de la vie économique dans le monde rural, chaque village et chaque chefferie possède son marché. La commercialisation des vivres pour l'approvisionnement des villes apparaît ainsi comme un facteur incontestable d'enrichissement dans la mesure où des revenus importants sont distribués aux paysans et de nombreux petits emplois sont induits par ce trafic. Il est donc important de comprendre le mode de fonctionnement de cette organisation commerciale et d'en connaître les acteurs.

17 Plus que les autres groupes ethniques du Cameroun, il est incontestable que les Bamiléké, paysans pour la grande majorité, ont su pratiquer les activités commerciales et ont conquis sur l'ensemble du Cameroun une position prépondérante. L'explication doit être recherchée dans les traditions sociales et le mode de fonctionnement d'un groupe qui incite l'individu à une promotion sociale liée à la réussite économique. La passion des Bamiléké vis-à-vis de l'argent, et le souci d'accumulation en vue d'investir trouvent un support efficace dans les institutions sociales et plus particulièrement dans les associations à caractère financier (tontines) qui facilitent l'épargne (Wamier, 1993 : 80-86). Il convient toutefois de remarquer que presque toutes les tentatives pour organiser ce commerce ont échoué, contrastant paradoxalement avec la réussite de l'Union Centrale des Coopératives Agricoles de l'Ouest (UCCAO) pour le café Arabica (Courade, 1991).

18 Ce qui témoigne une fois de plus de l'importance de la ville et des citadins dans l'organisation de la commercialisation des produits vivriers. En réalité le problème se pose en terme de collecte sur les marchés ruraux, de transport et de stockage et de distribution dans les centres urbains.

19 Parmi les acteurs du commerce des vivres sur les marchés de Douala on peut distinguer les catégories suivantes :

- les grossistes, essentiellement des hommes, qui disposent des magasins de stockages tandis que pour d'autres, la vente se fait à bord des camions ou des camionnettes ;

- les semi-grossistes ;

- les vendeurs au détail, qui sont surtout des femmes, dont le financement initial provient souvent des fonds propres familiaux, du capital décès ou des indemnités de licenciement du conjoint, d'une épargne mobilisée à travers les tontines ou du soutien d'une grossiste qui donne la marchandise à crédit. Les fonds de roulement sont modestes entre 5000 et 50000 FCFA.

20 Si la limite est assez floue entre commerce de gros, de demi-gros, et de détail, le meilleur critère pour délimiter les différents niveaux est l'unité de mesure utilisée lors de la vente. Les unités utilisées pour la vente au détail sont le verre, le gobelet, la pièce, le petit tas ou le seau, et au niveau du demi-gros, le sac, le fût, la caisse, le régime.

21 Dans le cadre de cet article nous avons volontairement restreint l'analyse à trois catégories d'acteurs : les transporteurs-commerçants, les commerçants-grossistes et les détaillantes. Les transporteurs-commerçants sont propriétaires de camions qui résident à Douala ou dans les villes de collecte : Bafoussam, Dschang, Mbouda, Foumbot ou Nkongsamba pour le circuit ouest qui ravitaille les marchés de Douala en maïs, macabo haricot, banane plantain, pommes de terre, cultures maraîchères et fruits ; Ngaoudéré, Garoua et Maroua pour le circuit nord dont les ventes portent essentiellement sur l'oignon et l'arachide. Ils ne se contentent pas de louer leurs véhicules à des tiers mais exercent également dans la commercialisation depuis la collecte dans les zones de production jusqu'à la distribution sur les marchés urbains. 
Dans l'approvisionnement et la distribution alimentaire des métropoles camerounaises, les transporteurs-commerçants sont en situation de concurrence avec les commerçants-grossistes qui eux n'ont pas de moyen de transport, mais louent individuellement ou de façon collective camions ou camionnettes pour l'acheminement des marchandises sur les marchés urbains. Ces deux catégories d'acteurs sont les principaux agents de liaison et de connexion entre la ville et le village, entre les ruraux et les citadins. Ils interposent entre le producteur et le marché urbain plusieurs types d'intermédiaires. D'une part les petits colporteurs qui vont à pied dans les marchés difficiles $d$ 'accès pour acheter à des prix très faibles des produits pour les revendre avec une marge modique sur des marchés plus accessibles aux grossistes, et d'autre part les petits collecteurs ruraux et les pisteurs qui les informent sur la disponibilité des stocks. Les acheteurs-groupeurs parcourent plusieurs petits marchés ruraux pour collecter à bas prix les produits vivriers ou animaux. En plus de ces partenaires, les commerçants-transporteurs et les commerçants-grossistes ont sous leur dépendance directe un ou plusieurs employés (chauffeurs, convoyeurs et hommes de confiance) dont le nombre varie selon le niveau de l'activité. Sur le marché vivrier de Douala, le commerçant travaille en collaboration avec des aides-vendeurs et des veilleurs de nuits qu'il faut ménager à chaque voyage.

Si, de manière générale, aucun contrat écrit ne lie le commerçant à ses différents partenaires (colporteurs, collecteurs, pisteurs, acheteurs-groupeurs) ou à ses employés (chauffeurs, convoyeurs, aides vendeur), il existe entre les uns et les autres une certaine complicité et un niveau de confiance qui dépend de l'appréciation que le commerçant fait du sérieux et du dynamisme des partenaires qu'il met très souvent en concurrence. Partenaires et employés sont recrutés soit en fonction de leurs appartenances au même groupes ethniques, soit en raison de leur connaissance des producteurs ruraux, des marchés, des demi-grossistes et des détaillantes. Les rapports ne sont plus seulement commerciaux mais davantage sociaux : expédition des courriers aux parents et amis en ville, achats des médicaments et des produits de luxe, hébergement temporaire des enfants pendant la première année de leur scolarité en ville en faveur des partenaires ruraux, consultation des tradipraticiens, contrôle des plantations ou des travaux de construction, organisation des funérailles et commissions diverses auprès des parents ou des autorités traditionnelles au village au profit des commerçants-citadins. Plus que des biens, les acteurs échangent ainsi des modèles de comportement, des complémentarités fonctionnelles allant au-delà du seul échange économique et parfois même des relations sociales, ce qui fait la spécificité du mode d'articulation paysan par rapport au mode d'intégration purement capitaliste (Minvielle, 1999).

23 Les Bamiléké ont mis au service de l'approvisionnement des villes en denrées alimentaire fraîches une tradition commerciale ancienne expérimentée jadis dans le commerce de l'ivoire et de l'huile de palme avec les régions forestières et côtières, dans le commerce de la kola avec le nord musulman, ou le commerce intra-régional de porcs, de chèvres, de condiments et de vin de raphia. L'innovation aujourd'hui par rapport à la période coloniale et au cours des premières années d'indépendance, c'est l'apparition et la multiplication d'une nouvelle catégorie d'interlocuteurs : polices et gendarmes officiellement chargés de la sécurité routière. Leur comportement et les rapports qu'ils entretiennent avec les commerçants influent sur bien des aspects de la commercialisation des denrées alimentaires (durée de voyage, qualité et prix des vivres sur les marchés urbains). Le calcul des coûts réels de commercialisation des produits 
vivriers et maraîchers entre les campagnes et les villes camerounaises doit prendre en compte non seulement les frais de location de véhicules, les diverses transactions sur les marchés de brousse et le prix du carburant en augmentation permanente, mais également les charges fiscales et para-légales que doivent supporter les transporteurs. En 1995, la structure des taxes est la suivante :

- 80000 FCFA de charge informelle entre Foumbot et Douala ;

- 69000 FCFA de taxe légale et officielle pour un chargement à Bafoussam à destination du Gabon ;

- 3500000 FCFA de taxe para-légale sur le même trajet ; 450000 entre Foumbot et Libreville la capitale du Gabon (Hatcheu, 1993). Rien malheureusement ne permet d'entrevoir la moindre amélioration aujourd'hui.

L'explication socio-anthropologique de l'omniprésence, la para-fiscalité doit être recherchée dans la corruption généralisée qui caractérise la société camerounaise. Le transporteur sait qu'il doit donner "la kola du chef" qu'il soit en règle ou non avec la législation en vigueur. Le dossier que "les mange mil" demandent, sous prétexte de contrôle, ne sert qu'à dissimuler le billet de 1000 FCFA qui tient lieu de kola. Les négociations et les marchandages éventuelles portent non pas sur l'authenticité ou la régularité de telle ou telle pièce de véhicule, moins encore sur la qualité, l'origine ou la destination du produit, mais sur la "dimension de la kola du chef". Autant le "mange mil" (gendarme, policier ou agent de bureau) est conscient de la complicité et du soutien de sa hiérarchie dans ces pratiques, autant le commerçant et le transporteur sait qu'il a intérêt à jouer serrer pour gagner en temps et en argent, surtout s'il s'agit des produits périssables comme les tomates et les fruits, et s'il doit arriver sur le marché de Lagos, Deido ou Madagascar pour honorer le rendez-vous matinal avec ses clients.

Les stratégies des acteurs et les rapports sociaux dans l'acte de vente

Les bayam sellam hommes cherchent des marchés éloignés aussi bien pour l'approvisionnement que pour la vente. Ainsi s'explique que Douala ne soit pas le seul lieu de vente. Pour réduire l'offre et augmenter les marges ; le commerçant bamiléké va à la conquête des marchés les plus éloignés, que ce soit à l'intérieur du pays (Yaoundé, Ngaoundéré, Garoua, Maroua) ou dans les pays voisins (Gabon, Tchad ou République Centrafricaine, et même certaines capitales occidentales). Pour s'assurer les bas prix à l'achat, ils bravent les pistes souvent mal entretenus et paient au prix fort la location des camions pour acheter dans les villages les plus reculés à des producteurs appauvris et peu ou pas du tout informés sur les prix et qui n'ont d'autre choix que de brader leur production pour payer la scolarité des enfants, acheter les médicaments ou consulter enfin le guérisseur.

Les bayam-sellam femmes ou revendeuses alimentent les marchés des centres urbains situés dans les zones de production. Elles dominent et maitrisent toute la distribution alimentaire sur les marchés vivriers de Douala (marché central, marché Madagascar, marché Deido pour les plus grands). Elles se sont imposées sur tous les petits marchés de rue de la capitale économique camerounaise (marché Koulouloum, marché de la Gare, petit marché du Rond point terminus au carrefour Saint Michel, etc.). A peine si ces "mamans", la cinquantaine bien entamée pour les trois quart des trente six revendeuses que nous avons pu dénombrer sur le petit marché du Rond point terminus au carrefour Saint Michel ont déjà entendu parler de la balance sous quelque forme que ce soit. Elles vendent en tas, en boîte, en verre, en seau, en cuvette ou à l'unité. Le choix 
de ces unités de mesure s'explique par leur practicabilité, mais l'explication par la pauvreté et la misère est de loin la plus plausible. Le fonds de roulement est maigre voire ridicule. Chez les vendeuses de rue il atteint rarement 20000 FCFA. Très souvent elles essayent de détourner la ration alimentaire dans l'espoir de dégager en fin de semaine un hypothétique bénéfice qui permettra de cotiser à la tontine dominicale (entre 500 et 1000 FCFA). Dans un contexte de forte croissance urbaine et de désorganisation des pouvoirs publics, qui pénalise les pauvres et invente chaque jour de nouvelles difficultés pour de nouveaux citadins plus dépourvus que jamais, et plus dépendants du village aujourd'hui qu'hier lorsqu'ils vivaient encore dans un milieu rural, la "maman" s'accroche pour ne pas disparaître économiquement, socialement, culturellement. Elles n'attendent rien ou presque de l'État si non le harcèlement de la police et le racket des vendeurs de ticket de marché se replaçant instinctivement dans un mode de fonctionnement familier, par un double réflexe de survie et de groupe. Elles travaillent essentiellement dans une logique de survie.

Une partie de leur marge provient d'un jeu subtil dans la façon de mesurer les quantités à l'achat et à la vente. En effet, la contenance des mesures locales (seaux et sacs) varie en fonction de la disposition des tubercules (macabo, pomme de terre, patate, banane poyo) dans le sac ou dans le seau. Ainsi s'explique la vigilance de ces vendeuses lors des opérations de vente et leur empressement à transvaser la marchandise une fois que le prix est arrêté après des négociations bien serrées. C'est alors qu'intervient le cadeau que l'acheteur réclame et exige et dont la vendeuse se fait le plaisir et l'honneur de donner. La quantité du cadeau varie en fonction de la nature du produit (une pincée de sel, quelques maigres plantains, un ou deux petits macabos ou des pommes de terre, quelque grains d'arachide ou de haricot) geste limité mais plein de signification et de symbole. Il serait simpliste de croire qu'il s'agit pour l'acheteur de compenser une perte et pour la vendeuse de combler le trop perçu. En effet, la logique du cadeau est loin d'être commerciale. Elle est sociale et rend compte de la nature des rapports sociaux entre la vendeuse et l'acheteur. Elle est révélatrice de la considération que ces partenaires ont l'un pour l'autre.

Le cadeau révèle un caractère symbolique, mais remplit une fonction psychologique irremplaçable dans la fidélisation de la clientèle. Les relations sociales sont encastrées dans les relations économiques. Le "si tu ne me mets pas le cadeau, c'est la dernière fois que j'achète chez toi" est une menace grave et l'expression d'une frustration que la vendeuse prend très au sérieux. Si les multinationales européennes et américaines engagent d'importants capitaux pour leurs campagnes de publicité, la bayam sellam camerounaise manie le geste et la parole qui rassurent et réconfortent l'acheteur. Comme le précise Kadidja Kane Devautour (1997) "ces pratiques trouvent leurs fondements, non seulement dans la précarité économique, mais surtout dans des logiques sociales récurrentes et dans des jeux de rôles auxquels chacun participe. Cette précarité et ces jeux de rôles expliquent l'échec des essais de réglementation parce qu'ils légitiment l'alliance naturelle de l'acheteur, du vendeur et de l'administrateur, cette alliance dans laquelle l'intérêt se substitue bien souvent à la responsabilité et dans laquelle la confusion de ces intérêts devient tellement naturelle que certains administrateurs, en toute bonne foi, ne se demandent même plus 'comment réglementer ?' mais 'à quoi bon réglementer?"'. Ces pratiques ont d'autant plus d'avenir qu'elles renouvellent la tradition africaine.

Les rapports avec les institutions financières 
l'étude des dossiers de demande de crédit et des processus d'emprunt, discrimination, ségrégation et clientélisme qui dissuadent les peu scolarisés, sélectivité des prêts, ce qui explique la méfiance des entrepreneurs et renforce la confiance et l'attachement aux structures traditionnelles de financement que sont les tontines.

L'accès au crédit auprès des institutions financières formelles est impossible pour ces acteurs économiques. L'indifférence à l'égard de ces institutions a progressivement cédé la place à la méfiance et au mépris quand la crise économique et la restructuration du secteur bancaire ont mis à nu l'ampleur du laxisme et de la corruption qui a pendant longtemps caractérisé la gestion des banques camerounaises. Si les causes de la faillite de la BIAO-Cameroun d'abord et de la BIAO-Méridian Bank ensuite sont exogènes, la récente banqueroute de l'ex-Crédit Agricole a eu le mérite de convaincre les transporteurs et les commerçants camerounais à rompre avec les institutions financières trop dépendantes de l'État. 25 Août 1992 pour combler les lacunes n'ont pas encore eu le temps de faire la preuve de leur sérieux et de leur crédibilité. Aussi, pour les nombreux transporteurs et commerçants, les structures traditionnelles de financement restent les plus viables et les plus accessibles. L'investissement de l'épargne individuel est la première source de financement de la phase de décollage. Le développement des cultures maraîchères dans la région de Fombot et l'amélioration des voies de communication routières entre Douala et Yaoundé ont permis des investissements, dans le domaine agricole, souvent complétés par l'élevage de la volaille et du porc. Le point de départ de l'accumulation est constitué par une activité lucrative susceptible d'autoriser la participation à une tontine. La commercialisation et/ou le transport des vivres est une étape dans la carrière, chaque étape servant de tremplin pour le passage à l'étape suivante. Tous ont épargné sur leur revenu et capitalisé relations et savoir-faire acquis au cours de la période préalable à la création de leur entreprise. L'accumulation pratiquée par l'entrepreneur bamiléké se fonde sur l'épargne régulière et soutenue, l'investissement et le verrouillage efficace de désinvestissement (Warnier, 1993).

Plus que tous les autres opérateurs, les commerçants et les transporteurs bamiléké ont trouvé dans la tontine un merveilleux outil de capitalisation. Ils affrontent ainsi des situations objectivement diverses avec des armes qu'ils puisent dans la civilisation de la région des hautes terre de l'ouest du Cameroun. Cette civilisation considère comme légitimes les inégalités sociales, la hiérarchie et, partant, l'accumulation.

Les recrutements dans les tontines d'affaires tiennent à la fois compte des liens de parenté et de l'origine géographique, du niveau et du secteur d'activité. La configuration la plus fréquente combine tontine de solidarité, avec les ressortissants du même village, tontine d'affaire de montant moyen avec les ressortissants de la province, ou une tontine d'affaire à taux de cotisation plus fort et de recrutement plus diversifié. La tontine présente au moins deux avantages fondamentaux : la rapidité de la mobilisation des montants importants dont dépend le succès de nombreuses entreprises et la constitution de réseau de relations d'affaire par le biais des contacts réguliers et approfondis favorisés par les réunions périodiques.

Dans l'approvisionnement et la distribution alimentaire dans les villes camerounaises, peut-on vraiment parler de réseau marchand de structure et d'organisation identique à ceux décrits par Emmanuel Grégoire et Pascal Labazée (1993) chez les commerçants 
d'Afrique de l'ouest. Quel est l'avenir sinon de ces réseaux du moins de ces commerçants dans une économie mondialisée?

Inquiétudes et incertitudes sur l'avenir des acteurs locaux et des réseaux dans la ville de demain

Le concept de réseau fait référence au principe de la multiplicité des relations et de la circulation des personnes, des biens, des capitaux, des idées et du savoir. Le réseau organise des différences. Le réseau fait émerger de l'anonymat des individus qui ont entre eux certaines affinités. Ces individus deviennent des relais vers d'autres individus. Le réseau se constitue en réorganisant l'offre et la demande. Le réseau suppose des acteurs susceptibles de mobiliser des relations sociales. Les liens sociaux noués demeurent conjoncturels et occasionnels. La connexité est la vraie nature du réseau. Il en tire toute sa puissance d'organisation. La connexité fait référence à la capacité du réseau de réaliser un grand nombre de liaisons, fussent-elles éphémères, entre des éléments ou des points qui en font partie. Dans l'organisation des réseaux règnent des mailles, des boucles, des redondances de toutes sortes.

Dés qu'un acteur social a en charge le fonctionnement d'un réseau, il doit maintenir un difficile équilibre entre la sauvegarde de toutes les possibilités offertes pur la complexité intrinsèque du réseau d'où provient le véritable pouvoir organisateur, et une forme minimale de gestion collective nécessaire pour inscrire le réseau dans l'espace. Si on admet cette définition on se rend bien compte que les transporteurs et les commerçants animent de véritables réseaux marchands constitués des individus partenaires qui, bien que s'ignorant, travaillent pour un objectif identique : l'expansion économique et le rayonnement social du patron.

Chez les Bamiléké qui exercent dans la commercialisation de vivres pour l'approvisionnement et la distribution alimentaire à Douala, l'individu-acteur est au centre de la problématique. On se situe alors dans la même perspective que Pierre Signoles (1999: 19), qui estime que "la plupart des recherches qui traitent des villes fournissent des éclairages sur les acteurs urbains dès lors qu'elles s'occupent de comprendre les processus de transformation, les dynamiques qui les animent, les pratiques qui les parcourent". L'approche par l'acteur fait avancer la réflexion au moins sur trois points :

- elle permet d'aborder la question de la régulation sociale dans la mesure où il n'y a pas de système social entièrement réglé. Ce qui pose la question de la légitimité des acteurs et de la légitimation de leur action ;

- l'approche en terme d'acteurs et de système d'action favorise un déplacement de l'objet de recherche. Le comportement a toujours un sens. Il est rationnel non pas par rapport à des objectifs mais par rapport à des opportunités et par rapport aux comportements des autres. Ce comportement a toujours deux aspects : offensif par des saisies des opportunités pour améliorer sa position, et défensif dans le cadre du maintien et de l'élargissement de sa puissance économique et de son assise sociale. Dans ce contexte, la stratégie apparaît alors comme le fondement des régularités des comportements observés empiriquement. Le concept de "stratégie" à la différence de la notion "d'objectif' oblige à chercher dans le contexte organisationnel la rationalité de l'acteur et à comprendre le construit organisationnel dans le construit de l'acteur;

- le fait de spécifier acteur public et acteur privé a une signification à la fois politique et idéologique dans la mesure où le rôle de l'acteur privé a été sous-estimé ou nié pendant de longues années. 

des acteurs. La seconde explication serait fondée sur les traditions mercantiles et les clivages entre les notables et les cadets bamiléké. L'entrepreneur est un agent qui opère en fonction des contraintes et des possibilités qui sont déjà là, et la civilisation informe son action en lui fournissant les cadres de sa perception, les objets de son désir et de multiples répertoires de conduite (ibid: 251). L'acteur obéit à des logiques, son comportement étant façonné par des traditions historiques et il fait appel à des discours, des registres ou des répertoires. Ces commerçants sont des agents qui par des choix stratégiques dessinent des trajectoires susceptibles de se joindre à d'autres trajectoires voisines pouvant aboutir à une action collective, à l'émergence des communautés des sociétés, d'organisations, et qui constituent le champ de toute action ultérieure. C'est ainsi qu'il traite la trajectoire des entrepreneurs qui, d'une position initiale de cadets, opèrent le choix migratoire, le choix du type d'emploi et de l'épargne, le choix matrimonial, etc. Cette trajectoire se combine à d'autres dans la création des tontines du village en ville, ou de la chefferie réinventée.

41 Les choix opérés par les agents le sont toujours en situation, et ils dépendent de la manière dont l'agent perçoit la situation, c'est-à-dire des cadres de la perception ainsi que les objectifs culturellement valorisés et acceptables. En d'autres termes, l'analyse des choix individuels doit prendre en compte les faits de civilisation inscrits en longue durée. Ce faisant ils intègrent de nouveaux réseaux "dont chacun est porteur de solidarité nouvelle" (Olivier de Sardan, 1996). Il s'agit ici non seulement des tontines du village ou des départements d'origine, mais de plus en plus des associations des anciens de tel collège ou lycée, des anciens de telle promotion de faculté ou de grande école, des anciens joueurs de telle équipe de football, des enfants de tel quartier d'une grande ville (New-bell à Douala, par exemple). 
42 Dans l'organisation et le fonctionnement des réseaux marchands, la notion de capital relationnel parait fondamentale. L'accumulation d'un type d'individu est corrélée à la détention de trois types de capital: le capital social, le capital religieux, le capital relationnel au sein de l'État. Outre la détention du capital économique, le clientélisme social, religieux ou étatique semblent être des éléments fondamentaux pour réussir dans les affaires. C'est en prenant en compte ces logiques des acteurs qui peuplent les réseaux marchands africains que le libéralisme parviendrait à éviter les incohérences et les impasses. Les politiques mises en œuvres dans le cadre des PAS ont mal apprécié la flexibilité des marchés, leur fonctionnement et le comportement des agents économiques soumis à des obligations diverses et à des rapports de pouvoir qui peuvent entraver le déroulement des activités et qui les font apparaître au regard du monde extérieur comme irrationnel.

On peut se servir de la grille d'analyse que propose Pascal Labazée (1991) qui distingue plusieurs modèles d'accumulation chez les entrepreneurs africains : le premier modèle d'accumulation concerne un groupe de promoteurs qui, venant de la fonction publique, occupait des postes de responsabilité au sein de l'appareil administratif. Le second concerne les négociants dont les affaires connurent à l'Indépendance des mutations considérables. Par convention Labazée appelle commerçants de "première génération" les négociants qui ont commencé leur carrière avant les indépendances puis accompli leur reconversion. La deuxième génération de commerçants est celle qui s'affirme aujourd'hui avec une scolarité bien souvent orientée vers la maîtrise des techniques de gestion et de comptabilité. Elle recourt aux techniques financières, se spécialise dans une gamme de produits, diversifie les placements. Autant de signe d'une refonte des pratiques du négoce et d'une rupture des représentations de l'entreprise et du profit.

44 L'avenir de la nouvelle génération d'entrepreneurs africains passe par leur capacité à réinventer perpétuellement les répertoires, par les innovations et les emprunts d'éléments nouveaux que lui impose l'évolution technique et technologique dans tous les secteurs d'activités d'une part, et par l'abandon de certaines conduites désuètes inhérentes à sa culture et à sa tradition d'autre part. L'intégration des entrepreneurs africains dans les grands courants des échanges mondiaux ou leurs aptitudes à se positionner comme des partenaires crédibles et sérieux des multinationales occidentales passe par leur capacité à intégrer dans les pratiques de la tontine les apports et les contraintes de la gestion rigoureuse des banques occidentales. Il faut alors réaliser un savant dosage entre les dépenses ostentatoires pour les funérailles, les investissements de prestige dans la construction de gigantesques résidences de campagne, le financement coûteux des pèlerinages à la Mecque et le soutien financier des activités plus rentables et des partenaires en quête de soutien. Il faut combiner avantageusement les contraintes imposées par la tradition ou la religion, les exigences de la vie sociale et le nécessaire développement des affaires.

45 La connexion à la diaspora africaine en Europe, aux États-Unis et de plus en plus en Asie et en Australie apparaît à plusieurs égards comme une chance supplémentaire pour les commerçants africains. Cette diaspora constitue des maillons essentiels et indispensables à l'intégration dans les grands courants d'échanges que la mondialisation fait miroiter à nos yeux. C'est peut-être la voie à suivre pour que les nouveaux hommes d'affaires africains qui plus que leurs ânés deviennent ces partenaires que les organismes internationaux et les multinationales désirent voir fleurir en Afrique. De par leur capacité à concilier la religion, la tradition et certains 
aspects de la modernité et à séparer l'économique du social, ils sont finalement proches des normes occidentales. Dans ce contexte, religion et tradition ne sont pas des freins au commerce. Au contraire, elles définissent un cadre conceptuel parfaitement adapté aux exigences des affaires, une éthique et des règles de conduite.

Dans ce processus de connexion du village à la ville qui constitue en réalité une étape vers la connexion des marchés africains de Château Rouge ${ }^{1}$, européens et américains, il reste à redéfinir la place de l'État, acteur et partenaire certes incontournable mais bien encombrant dans le type de fonctionnement que lui ont imposé les politiques d'ajustement structurel. On a besoin d'un État capable d'établir un environnement transparent, sécurisant et stable, d'un État capable dans le cadre de la démocratisation et de la décentralisation de joindre son action à celle des entrepreneurs pour faciliter l'accès au crédit, la circulation des marchandises entre la campagne et la ville, et améliorer la gestion, non seulement des marchés de gros, mais de la chose publique tout court. C'est l'objectif majeur recherché à travers la bonne gouvernance que les bailleurs de fonds et les partenaires occidentaux exigent des États africains face à "ampleur de la corruption. Mais les experts de la Banque mondiale et du Fonds monétaire international n'ont-ils pas Sous-estimé les effets pervers des politiques d'ajustement structurel? La dévaluation du FCFA a renchéri le prix des céréales importées et redonné une chance aux productions vivrières locales sur les marchés urbains. Toutefois la demande alimentaire a été réorientée vers les produits nutritionnellement pauvres lorsque les villes sont frappées par le chômage et la réduction des revenus dus au PAS (cas de la banane poyo sur le marché de Madagascar à Douala). Mais c'est par naïveté et par mesquinerie que ceux-là même qui ont imposé de façon simultanée dévaluation du FCFA, surtaxation de l'activité économique, baisse drastique des salaires, licenciements et compressions massifs dans le public et le privé, se plaignent et se lamentent que le Cameroun reste au hit-parade des pays les plus corrompus de la planète.

47 Le sous paiement des fonctionnaires et des agents de l'État et l'obligation d'entretenir une clientèle de plus en plus nombreuse et de plus en plus exigeante (famille étendue, parents et amis du village, camarades du Parti, associés et dépendants divers) fait de l'État une vache à lait, et de la chose public, un gâteau à partager. Il devient alors impérieux et vital pour l'entrepreneur d'étendre et de consolider ses réseaux de solidarité et de pression dans la plus haute sphère de l'administration.

En attendant de créer un État tutélaire qui constitue une composante de la société et avec lequel on noue des relations de par1enariat authentique et qui joue à fond son rôle de facilitateur et de régulateur de l'activité économique, en Afrique les politiques d'ajustement structurel ont progressivement façonné des "éléphants blancs que l'on dépèce" (on parle au Cameroun de partage et d'accès au gâteau national). Ici les entrepreneurs se sont construits une image qui varie en fonction des attentes.

Conclusion

49 Si le phénomène urbain constitue à la fois une réalité et un défi que l'Afrique doit relever au cours de ce troisième millénaire, les incompatibilités entre la quête de la sécurité alimentaire et certaines mesures prises dans le cadre des politiques d'ajustement structurel et l'acuité des problèmes fonciers ont rendu les villes encore plus dépendantes des campagnes. Au-delà de l'approvisionnement des villes par les campagnes et de la commercialisation des vivres les acteurs développent des comportements et des pratiques qui obéissent à des logiques. En mettant à leur service 
et en concurrence des partenaires dispersés dans le temps et dans l'espace entre les aires d'approvisionnement et les marchés urbains, commerçants et transporteurs animent de véritables réseaux marchands. En attendant que la diaspora africaine en Occident parachève l'intégration de la nouvelle génération des commerçants africains aux grands courants des échanges internationaux, les revendeuses qui contrôlent la distribution alimentaire dans nos villes puisent dans la tradition africaine des pratiques qui leurs permettent à travers le cadeau de fidéliser la clientèle et de trouver dans les structures traditionnelles les financements dont ils sont exclus auprès des banques classiques.

Toutefois, la commercialisation des vivres et l'approvisionnement des villes par les campagnes doit compter avec la pratique de la corruption qui dans le cas particulier du Cameroun s'est généralisée à la faveur de la réduction drastique des revenus et du pouvoir d'achat du citoyen camerounais. C'est qu'au Cameroun, l'État régulateur et facilitateur de l'activité économique est encore à inventer. Pour le plus haut commis de l'administration comme pour l'agent de police ou le gendarme chargé de la sécurité routière, le commerçant et le transporteur est loin d'être un usager, moins encore un citoyen susceptible de prétendre à un quelconque droit. Il est d'abord et avant tout un client qui doit à chacun des multiples barrages payer la cola du chef. Le débat sur le rôle de l'État dans les échanges villes/campagnes débouche ainsi sur le destin des réseaux marchands africains dans le processus de la mondialisation de l'économie internationale.

\section{BIBLIOGRAPHIE}

Chaleard J.L., 1996 Temps des villes temps des vivres : l'essor du vivrier marchand en Côte d'Ivoire. Paris : Karthala.

Courade G., 1985. "Villes/campagnes : les liaisons dangereuses". in Bricas et al., Nourrir la ville en Afrique subsaharienne. Paris, L'Harmattan : 67-81.

Courade G., 1999. "Les paysanneries du sud et la libéralisation des échanges". in Haubert M. (ed.), L'avenir des paysans : les mutations de l'agricultures familiales dans les pays du sud. Paris, Editions Tiers-Monde IEDES : 61-75.

Courade G., Eloundou-Enyegue P., Grangeret I., 1991. "L'Union Centrale des Coopératives Agricoles de l'Ouest du Cameroun (UCCAO) : de l'entreprise commerciale à l'organisation paysanne". Revue Tiers-Monde. XXXII, nº 128 : 887-899.

Dongmo J.L. et al., 1976. Recherches sur l'approvisionnement des villes. Mémoires du centre d'étude de géographie tropicale du CNRS, collection la croissance urbaine dans les pays tropicaux, Paris.

Goossens F., Minten B., Tollens E., 1994, Nourrir Kinshasa : L'approvisionnement local d'une métropole africaine. Paris : L'Harmattan.

Gregoire E., Labazée P., 1993. Grands commerçants d'Afrique de l'Ouest : logiques et pratiques d'un groupe d'homme d'affaires contemporains. Paris : Karthala. 
Hatcheu Tchawé E., 1998. Une évaluation de l'impact de la dévaluation du FCFA sur la production agricole dans la province de l'Ouest du Cameroun. Les dossiers du JCAD n², Dschang.

Kadidja Kane Devautour, 1997. Logiques sociales et pratiques informelles et leurs implications pour les programmes d'appui aux SADA des villes d'Afrique francophone, le cas de la Mauritanie. Rome : FAO.

Labazée P., 1991. "Un terrain anthropologique à explorer : l'entreprise africaine". Cahiers d'études africaines, 124, XXI-4 : 533-552.

Mainet G., 1986. Douala, Croissance et servitude. Paris : L'Harmattan.

Olivier de Sardan J.P., 1996. "L'économie morale de la corruption en Afrique", Politique Africaine, $63: 97-116$

Minvielle J.P., 1999. "L'articulation des paysans au marché". in Haubert M. (ed.), L'avenir des paysans les mutations de l'agricultures familiales dans les pays du sud. Paris, Editions Tiers-Monde IEDES : 107-121.

Roubaud F., 1994. "Le modèle de développement camerounais 1965-1990 : de la croissance équilibrée à la crise structurelle". in Courade G. (ed.), Le village Camerounais à l'heure de l'ajustement., Paris, Karthala : 40- 49.

Signoles P., 1999. "Acteurs publics et acteurs privés dans le développement des villes arabes". in Signoles P., Galila El Kadi, Rachid Sidi Boumedine (eds.), L'urbain dans le monde arabe, politiques, instruments et acteurs. Paris, CNRS Editions : 19-53.

Schilter C., 1991. L'agriculture urbaine à Lomé. Paris : IUED-Karthala.

Vennetier P., 1991. Les villes en Afrique tropicales. Paris : Masson.

Warnier J.P., 1993. L'esprit d'entreprise au Cameroun. Paris : Karthala.

\section{NOTES}

1.Situé dans le $18^{\mathrm{e}}$ arrondissement de Paris, le marché de Château Rouge est le marché africain de la capitale française.

\section{AUTEUR}

\section{HATCHEU EMIL TCHAWE}

Université de Dschang, BP 394. Dschang (Cameroun) Tél. (+237) 451447 Fax, (+237) 452068 . 